The

American Journal

of Drug and

Alcohol Abuse

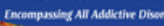

The American Journal of Drug and Alcohol Abuse

Encompassing All Addictive Disorders

\title{
Psychometric properties of the Persian version of the Time to Relapse Questionnaire (TRQ) in substance use disorder
}

\section{Maryam Khazaee-pool PhD, Minoo Moridi MSc, Koen Ponnet PhD, Nigel Turner PhD \& Tahereh Pashaei PhD}

To cite this article: Maryam Khazaee-pool PhD, Minoo Moridi MSc, Koen Ponnet PhD, Nigel Turner PhD \& Tahereh Pashaei PhD (2016) Psychometric properties of the Persian version of the Time to Relapse Questionnaire (TRQ) in substance use disorder, The American Journal of Drug and Alcohol Abuse, 42:6, 682-688, DOI: 10.3109/00952990.2016.1172593

To link to this article: http://dx.doi.org/10.3109/00952990.2016.1172593

曲 Published online: 10 Jun 2016.

Submit your article to this journal $₫$

Щ Article views: 27

Q View related articles ¿

View Crossmark data $\nearrow$ 


\title{
Psychometric properties of the Persian version of the Time to Relapse Questionnaire (TRQ) in substance use disorder
}

\author{
Maryam Khazaee-pool, $\mathrm{PhD}^{\mathrm{a}}$, Minoo Moridi, MSc ${ }^{\mathrm{b}}$, Koen Ponnet, $\mathrm{PhD}^{\mathrm{c}, \mathrm{d}, \mathrm{e}}$, Nigel Turner, PhD ${ }^{\mathrm{f}, \mathrm{g}, \mathrm{h}}$, \\ and Tahereh Pashaei, $\mathrm{PhD}^{\mathrm{b}}$
}

\begin{abstract}
${ }^{a}$ Department of Health Education and Promotion, School of Health, Zanjan University of Medical Sciences, Zanjan, Iran; ${ }^{b}$ Department of Public Health, School of Health, Kurdistan University of Medical Sciences, Sanandaj, Iran; 'Faculty of Social Sciences, Faculty of Law,

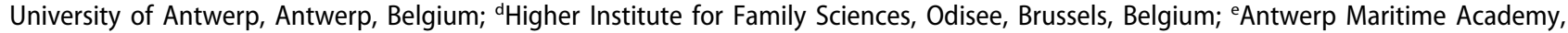
Antwerp, Belgium; 'Social Epidemiological Research, Centre for Addiction and Mental Health, Toronto, Canada; ${ }^{9}$ Dalla Lana School of Public Health, University of Toronto, Toronto, Canada; ${ }^{h}$ Social Epidemiological Research \& Problem Gambling Institute of Ontario, Centre for Addiction and Mental Health, Toronto, Canada
\end{abstract}

\begin{abstract}
Background: Predicting time to relapse provides an opportunity for the development of relapse prevention interventions in drug users. Objectives: The aim of the present study was to describe the development of the Persian version of the 9-item Time to Relapse Questionnaire (TRQ) and to evaluate its psychometric properties in an Iranian sample of treatment-seeking individuals with substance dependence $(n=150)$. Methods: The forward-backward method was used to translate the TRQ scale from English into Persian. After linguistic validation and a pilot check, a cross-sectional study was performed, and psychometric properties of the Iranian version of the questionnaire were assessed. The reliability was evaluated by Cronbach's alpha and test-retest analyses. In addition, the factor structure of the scale was extracted by applying confirmatory factor analysis. Results: The mean age of participants was $40.52(S D=11.30)$ years. The mean scores for the content validity index $(\mathrm{CVI})$ and the content validity ratio (CVR) were 0.93 and 0.81 , respectively. A confirmatory factor analysis (CFA) demonstrated that the three-factor model of the TRQ was a good fit for the data and thus replicated the factor structure of the original English language TRQ. Cronbach's alpha presented good internal consistency (alpha $=0.76$ ), and test-retest reliability of the TRQ instrument with 2-week intervals was appropriate (ICC $=0.84$ ). Conclusion: The findings demonstrate that the Persian version of the TRQ is a reliable and valid scale for measuring time to relapse in Iranian drug users. The TRQ can be applied at the start of treatment so that clinical interventions can be targeted toward the different relapse styles.
\end{abstract}

\section{ARTICLE HISTORY}

Received 8 March 2015

Revised 24 March 2016

Accepted 28 March 2016

\section{KEYWORDS}

Psychometric properties; time to relapse; Persian version; Iran

\section{Introduction}

Drug addiction is a social, psychological, and economic problem in Iran (1). Iran has a long history of substance abuse, and the existing documents show that Iranian people have been familiar with the medical properties of opiates for centuries (2). In addition to opium as a traditional drug, other popular drugs include heroin, synthetic heroin (called Kerack), norgesic, and temgesic; more recently, methamphetamine use has also been identified as an upcoming drug problem in Iran (3). There are several reasons for the drug abuse problem in the country. First, Iran has a long border with Afghanistan, the largest producer of opium in the world (4). Second, Iran is located on a drug transportation route $(1,5,6)$. Consequently, it is estimated that at least 1.2 million people in Iran are currently addicted to drugs (7). Besides needing appropriate diagnostic and therapeutic instruments, this huge number of drug addicts would also benefit from treatment services (8). Many different types of drug use treatment are available in Iran, such as a short-term medical detoxification with clonidine or naltrexone, ultra-rapid detoxification, buprenorphine maintenance treatment, methadone maintenance treatment, opium tincture substitution therapy, naltrexone maintenance treatment, Narcotics Anonymous treatment centers, residential centers (camps), matrix model intensive outpatient programs, and therapeutic communities for methamphetamine use treatment (3). Additionally, relapse prevention programs are available in some private and public addiction treatment centers. Since addiction is identified as a chronic, relapsing disease, relapse is acknowledged as a major challenge for addiction therapists $(9,10)$. Despite the development of various treatments for addiction in Iran, a significant number of patients return to drug use behavior. For instance, in a study conducted by Mohammadpoorasl 
et al., the rate of relapse after six months of treatment was $64 \%$ (11), while a study by Shirinbayan (12) found that six-month retention in methadone maintenance treatment (MMT) was only $22.7 \%$. Other Iranian studies have determined a high rate of relapse (between $75 \%$ and $95 \%)(13,14)$. Since longer retention is related to the effectiveness of addiction treatment, a high rate of relapse can be identified as a barrier to drug addiction treatment $(15,16)$.

Therefore, relapse prevention interventions designed by therapists and tailored to the relapse risk of the individual patient are a priority. The results of one study on relapse prevention showed that relapse prevention programs reduced relapse in Iranian drug users (17). However, a major limitation in designing relapse prevention interventions is the limited availability of psychometric instruments to measure relapse written in the Persian language. Although several researchers have attempted to develop reliable and valid instruments to assess relapse in drug users (18-20), most of them suffer problems in assessing the time to relapse for alcohol and drug use. Therefore, Adinoff and colleagues designed the Time to Relapse Questionnaire (TRQ) to assess the time from one's initial thought of drug use to actual use (21). TRQ is a self-reported, clear, and reliable instrument in settings of addiction treatment, and it is useful for patients with previous experience of relapse. The main value of the questionnaire is that it may predict what patients will relapse without forewarning compared to those who will do so with a period of delay. This would provide an opportunity to target therapy toward a patient's specific relapse risk. To our knowledge, however, the predictive validity of this measure has not been empirically validated. A Persian version of the TRQ could be used to overcome the lack of a suitable instrument for designing relapse prevention intervention in Iran. Given the strengths of the TRQ, the aim of this study is to describe the development of a Persian version of the TRQ and evaluate the psychometric properties of its obtained scores in drug-dependent patients.

\section{Methods}

\section{The Time to Relapse Questionnaire}

The TRQ is a self-report measure that assesses relapse risk in the drug- and alcohol-dependent population, using time to relapse as the critical assessment item. The TRQ consists of nine items and three subscales. The Sudden Relapse subscale consists of three items and refers to a fast (e.g., a few minutes) relapse to drug use. The 3-item Short Delay Relapse subscale refers to the returning to substance use in a very short time (i.e., less than one day). The three items on the Long Delay Relapse subscale reflect major cognitive control and might show more doubt toward using drugs. Each item is rated on a 4-point Likert scale: $1=$ false, 2 = slightly true, $3=$ mainly true, and $4=$ very true. As such, the total score can range from 3 to 12 for each subscale. A score above 9 in a subscale (Sudden Relapse, Short Delay Relapse, or Long Delay Relapse) suggests that this subscale is the patient's main relapse style. Some examples of items within each of the subscales include: When I start using drugs again, it's not planned (Sudden Relapse), I crave for less than one day before I start using again (Short Delay Relapse), I plan for several days before I use(Long Delay Relapse).

\section{The Persian version of the TRQ}

Permission to translate the English version of the TRQ was obtained from the original author (personal communication). The standard forward-backward method was used to translate the TRQ from English to Persian (the Iranian language). For forward translation, two bilingual professional translators independently translated the items of the TRQ scale into Persian. Next, these translations were integrated into a provisional Persian version of the TRQ. Then, two other professional translators who had never seen the English version of the questionnaire translated the Persian version back into English, and an interim English version of the questionnaire was created. Thereafter, a professional team comprised of translators, psychologists, and epidemiologists compared the original version of the TRQ with the back-translated English version. After some cultural and linguistic adaptations, a preliminary Persian version of the TRQ questionnaire was prepared. This Persian version was tested in a pilot study with 20 users, and thereafter, the final Persian version of the TRQ scale was solidified and applied in the present study.

\section{Procedure and participants}

The present study used a cross-sectional psychometric design and was conducted in Sanandaj, a large city in Iran, during the period of May-December 2014. A group of substance-dependent patients referred to drop-in centers (DICs) and methadone maintenance treatment (MMT) programs were selected using a randomized sampling method. The DICs are run by local nongovernmental organizations (NGOs) offering therapies and psychosocial support and facilitating self-help groups. Inclusion criteria in this study were: (a) being dependent on drugs in accordance with the Diagnostic 
and Statistical Manual of Mental Disorders, Fifth Edition (DSM-5), (b) having a history of previous treatment and experience of relapse, and (c) having no history of psychiatric disease. According to Gable and Wolf (22), the necessary sample size for a study can be determined by the number of questionnaire items multiplied by 10 (in this study, $9 \times 10=90$ ). Thus, the sample size of 150 in the initial study was adequate for the analyses employed. A multistage cluster sampling method was used. Firstly, Sanandaj was separated into five areas: north, south, west, east, and central. All MMT programs and DICs located in these five areas were recognized. Then, for each area, four MMT programs and one DIC were randomly chosen, and six people were approached at each of them. Additionally, 40 substance-use-dependent patients from the MMT programs and DICs completed the questionnaire twice in a two-week interval. After an interview and a short explanation about the study, patients who agreed to participate in this study were asked to answer the Iranian version of the TRQ questionnaire. If participants were illiterate, the researcher read them the questions and recorded their answers.

The mean age of the participants was 40.52 years $(\mathrm{SD}=11.30)$. Most participants $(n=98$, or $65.3 \%)$ were illiterate or had only achieved a primary education level. Of the participants, $23.3 \%(n=35)$ were unemployed. Of the $76.7 \%$ of the sample who had a job, about $54.7 \%$ worked full time. Regarding marital status, about $26.0 \%$ were single, $58.7 \%$ were mar ried, and $15.3 \%$ were divorced. The demographic characteristics of the participants are presented in Table 1. Table 2 presents the drug-use patterns of the participants. Opium and opium juice, heroin, and amphetamine-based drugs were the main current drug of abuse. Although, $55.3 \%$ of the patients $(n=83)$ were polydrug abusers who used at least three substances in an indiscriminant way, $26 \%$ of the participants $(n=39)$ used only two substances. Furthermore, $18.7 \%$ of the patients $(n=$ 28) had a history of injection drug use. All patients had experienced previous treatment; the most common treatment goal was an abstinence regimen.

\section{Analytic strategy}

Psychometric properties of the Persian version of the TRQ questionnaire were evaluated by assessing the internal and test-retest reliabilities; determining the content, face, and construct validities; and by examining the factor structure.

\section{Reliability}

Cronbach's alpha coefficient was used as a measure to assess the internal consistency of the questionnaire. Values equal to or above 0.70 are often considered to be satisfactory. However, Fitzner (2007) considered a respectable reliability to be between 0.70 and 0.80 and a very good reliability to be between 0.80 and 0.90 (23). Furthermore, to evaluate the questionnaire's stability, test-retest reliability was measured using the intraclass correlation coefficient (ICC). The ICC is a measure for

Table 1. Demographic characteristics of the study sample $(n=150)$.

\begin{tabular}{|c|c|c|c|c|c|}
\hline & & Face validity sample $(n=10)$ & Pilot study sample $(n=20)$ & CFA sample $(n=150)$ & $\begin{array}{l}\text { Test-retest sample } \\
\qquad(n=40)\end{array}$ \\
\hline & & Number (\%) & Number (\%) & Number (\%) & Number (\%) \\
\hline \multicolumn{6}{|l|}{ Age (years) } \\
\hline & $30 \leq$ & $1(10.0)$ & $6(30.0)$ & $27(18.0)$ & $13(32.5)$ \\
\hline & $31-45$ & $2(20.0)$ & $7(35.0)$ & $80(53.3)$ & $21(52.8)$ \\
\hline & $46-60$ & $6(60.0)$ & $5(25.0)$ & $36(24.0)$ & $4(10.0)$ \\
\hline & 61 and above & $1(10.0)$ & $2(10.0)$ & $7(4.7)$ & $2(5.0)$ \\
\hline & Mean (SD) & $41.87(9.27)$ & $40.52(8.63)$ & $40.52(11)$ & $36(9.88)$ \\
\hline & Range & $18-65$ & $24-72$ & $19-78$ & $21-67$ \\
\hline \multicolumn{6}{|l|}{ Employment status } \\
\hline & Jobless & $2(20.0)$ & $6(30.0)$ & $35(23.3)$ & $9(22.5)$ \\
\hline & Full time & $6(60.0)$ & $5(25.0)$ & $82(54.7)$ & $25(62.5)$ \\
\hline & Part time & $2(20.0)$ & $9(45.0)$ & $33(22.0)$ & $6(15.0)$ \\
\hline \multicolumn{6}{|c|}{ Income (in Iranian Rial) } \\
\hline & $500.0000<$ & $4(40.0)$ & $14(70.0)$ & $90(60.0)$ & 19 (47.5) \\
\hline & $500.0000-1.000 .0000$ & $3(30.0)$ & $5(25.0)$ & $49(32.7)$ & $16(40.0)$ \\
\hline & $1.000 .0000>$ & $3(30.0)$ & $1(5.0)$ & $11(7.3)$ & $5(3.5)$ \\
\hline \multicolumn{6}{|l|}{ Educational status } \\
\hline & Primary & $3(30.0)$ & $12(60.0)$ & $98(65.3)$ & $20(50.0)$ \\
\hline & Secondary & $5(50.0)$ & $6(30.0)$ & $39(26.0)$ & $12(30.0)$ \\
\hline & Higher & $2(20.0)$ & $2(10.0)$ & $13(8.7)$ & $8(20.0)$ \\
\hline \multicolumn{6}{|l|}{ Marital status } \\
\hline & Divorced/widowed & $1(10.0)$ & $8(40.0)$ & $23(15.3)$ & $7(17.5)$ \\
\hline & Married & $6(60.0)$ & $7(35.0)$ & $88(58.7)$ & $16(40.0)$ \\
\hline & Single & $3(30.0)$ & $5(25.0)$ & $39(26.0)$ & $17(42.5)$ \\
\hline
\end{tabular}


Table 2. Drug-use patterns of the study sample $(n=150)$.

\begin{tabular}{llrr}
\hline Drugs used & & Number & Percent \\
\hline Type of drug & Opium & 62 & \\
& Heroin & 56 & 41.4 \\
& Crystal meth & 5 & 37.3 \\
& Crack & 2 & 3.3 \\
& Tramadol & 3 & 1.3 \\
& Methadone & 22 & 2.0 \\
Injection & & 28 & 14.7 \\
& Yes & 122 & 18.7 \\
& No & & 81.3 \\
\hline
\end{tabular}

estimating the reliability where the unit of analysis is the individual rating, whereas Cronbach's alpha is a measure for estimating reliability where the unit of analysis is the mean of all the ratings (24). ICC values equal to or higher than 0.40 are considered to be acceptable ( $r$ 's between 0.81 and 1.0 are excellent, between 0.61 and 0.80 are very good, between 0.41 and 0.60 are good, between 0.21 and 0.40 are fair, and between 0.00 and 0.20 are poor) (25).

\section{Validity}

The content, face, and construct validities of the Persian version of the TRQ questionnaire were evaluated in the following ways.

\section{Content validity}

Both qualitative and quantitative methods were used to assess the content validity of the questionnaire. In a qualitative step, an expert panel consisting of two psychometric experts and five health professionals in the fields of health education and promotion, psychology, addiction therapy, and social medicine evaluated the grammar, wording, item allocation, and scaling of the TRQ questionnaire. The content validity index (CVI) and the content validity ratio (CVR) were assessed in a quantitative phase. To determine the CVI, the specialists were invited to assess each item based on relevance, clarity, and simplicity using a 4-point Likert-type scale ranging from $1=$ not relevant, not simple, and not clear to $4=$ very relevant, very simple, and very clear. A CVI score of 0.80 or above for each item was considered to be acceptable (26). The necessity of the items was evaluated by calculating the CVR, by which experts evaluated items as essential, useful but not essential, or unessential (27) based on the Lawshe Table (28); items with a CVR score below 0.40 were assumed to be not acceptable.

\section{Face validity}

In order to assess the face validity, both quantitative and qualitative methods were used. A group of drug users with different demographic characteristics and drug addictions $(n=10)$ were asked to assess each item of the questionnaire on "ambiguity," "difficulty," and "relevance." Revisions were made based on the participants' recommendations. In addition, a similar group of participants $(n=10)$ was asked to assess the importance of each item on a 5-point Likert scale. We then calculated impact scores (frequency $\times$ importance) and considered an impact score of 1.5 or below to be unsuitable (29).

\section{Construct validity}

A confirmative factor analysis (CFA) was applied to determine the underlying constructs of the Iranian version of the TRQ questionnaire. The model fit of the CFA was evaluated according to several fit indices. We reported a $\chi^{2} / \underline{\mathrm{d} f}$ ratio. An $\chi^{2} / \underline{d} f$ ratio between $2: 1$ and 5:1 is required for an acceptable fit; however, values of less than 3:1 are preferred (Kline, 2005). In addition, we examined the Goodness of Fit Index (GFI), the Non-Normed Fit Index (NNFI), the Normed Fit Index (NFI), the Root Mean Square Error of Approximation (RMSEA), and the Standardized Root Mean Square Residual (SRMR) (30,31). The NNFI and the NFI range from 0 to 1.00 . Values of 0.95 or higher indicate that a model provides a good fit while a value of at least 0.90 indicates that the model provides an adequate fit. RMSEA values below 0.05 indicate a good model fit while values between 0.06 and 0.08 indicate an adequate fit. The SRMR is a standardized summary of the average covariance residuals (32); a relatively good model fit is indicated when the SRMR is less than 0.08 .

\section{Ethics}

It was made clear at the beginning of the study that the respondents were under no obligation to participate and that they were allowed to leave the study at any time. Furthermore, the purpose and procedure of the study were explained to all participants, and their written informed consent was obtained. Ethical approval of this study was given by the Medical Ethics Committee of Kurdistan University of Medical Sciences.

\section{Results}

\section{Reliability}

The internal reliability of the questionnaire was measured using Cronbach's alpha. All values were above acceptable thresholds, with alpha $=0.76$ for the total questionnaire and alpha's ranging between 0.72 and 0.79 for its subscales. Furthermore, the ICC was 0.85 for the total questionnaire, with ICC values ranging from 0.805 to 0.915 for the subscales, lending support to the stability of the scales. The findings are displayed in Table 3. 
Table 3. Reliability estimates for the TRQ subscales.

\begin{tabular}{lllcr}
\hline Factor & Name of factor & Number of items & Cronbach's $a$ & 0.79 \\
\hline 1 & Long Delay Relapse & 3 items $(3,5,9)$ & 0.72 & 0.915 \\
2 & Short Delay Relapse & 3 items $(2,6,7)$ & 0.78 & 0.823 \\
3 & Sudden Relapse & 3 items $(1,4,8)$ & 0.763 & 0.805 \\
Total & & 9 items & & 0.847 \\
\hline
\end{tabular}

\section{Content and face validity}

The CVI and CVR, two measures of content validity, were good $(\mathrm{CVI}=0.93$ and $\mathrm{CVR}=0.81)$. In order to examine the face validity of the (sub)scales, impact scores were measured. The values were equal to or above 1.5 (ranging from 1.5 to 4.7 ) for the items. So, all items were kept in this phase (see the Method section above).

\section{Construct validity}

The construct validity was tested using a confirmatory factor analysis conducted on the multi-item scale in order to identify whether the indicators adequately measured the three constructs. The factors were consistent with the original TRQ (Adinoff, Talmadge et al., 2010), with factor 1 (Sudden Relapse) including three items (items 1, 4, and 8); factor 2 (Long Delay Relapse) including three items (items 3,5, and 9); and factor 3 (Short Delay Relapse) including three items (items 2, 6, and 7). The model fit the data well, with $\chi^{2} / \mathrm{d} f=1.64$, RMSEA $=0.066(90 \% \mathrm{CI}=0.021-0.089), \mathrm{SRMR}=0.03$, $\mathrm{GFI}=0.94$, NNFI $=0.95$, and NFI $=0.98$. As shown in Figure 1, factor loadings ranged from 0.61 to 0.72 for the Short Delay Relapse construct, from 0.71 to 0.78 for the Sudden Relapse construct, and from 0.72 to 0.78 for the Long Delay Relapse construct.

\section{Discussion}

This study described the development of a Persian version of the Time to Relapse Questionnaire (TRQ) and its psychometric properties. The original English version of the TRQ was translated and adapted into Persian in order to fill the absence of a validated instrument to measure time to relapse in Iranian drug users. To the best of our knowledge, the Persian TRQ is the first translated version of the original English TRQ.

Consistent with the original English version of the TRQ (21), three factors were found: Sudden Relapse,

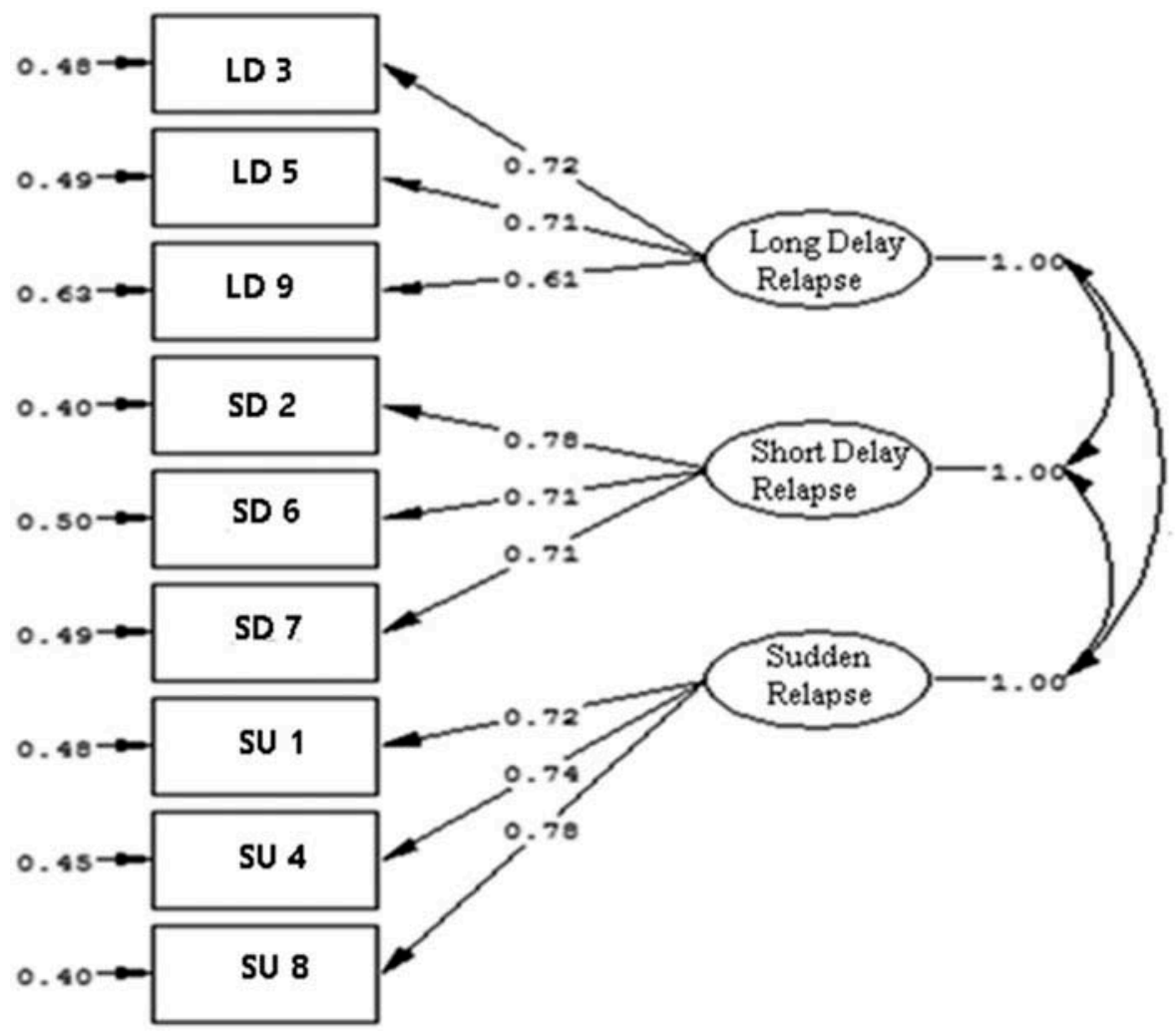

Figure 1. Confirmatory factor analysis of the TRQ. 
Short Delay Relapse, and Long Delay Relapse. Overall, the results demonstrated that the Persian version of the TRQ is valid and reliable for evaluating the time to relapse in drug users. Cronbach's alpha coefficient of the Persian TRQ was 0.76, indicating that the reliability was good. This finding is similar to the results reported by Adinoff and colleagues, the developers of the original English questionnaire. In their study, the authors reported a Cronbach's alpha of 0.61 for the full scale and between 0.64 and 0.75 for the subscales (21). Furthermore, in the present study, the ICC was 0.85, which was higher than that of the English version (2010). As such, it can be concluded that the Persian version of the TRQ has excellent reliability.

We observed three subscales in the confirmatory factor analysis, which were similar to those reported by Adinoff and colleagues. This may have been due to the drug-using features of the samples in both studies. The reason for high values of GFI, AGFI, and RMSEA might be that they could be impacted by sample size, which in this study was relatively satisfactory (33).

Items comprising the Sudden Relapse factor suggest a surprising and fast (a few minutes) return to drug use. There might be some important factors of desire, associated with the personal need, for a drug, like positive expectancies for drug use or lack of control over substance use. The Short Delay Relapse subscale refers to a return to substance use in a very short time (less than one day). Some interventions might therefore consist of methods like relaxing, doing hobbies, or talking with psychotherapists. Items related to the Long Delay Relapse subscale reflect major cognitive control and might show more doubt toward using drugs. Relapse might be more affected by certain factors, such as desire, ecological stressors, and positive mood conditions. Besides the aforementioned interventions, patients in this stage might have adequate time to see their psychotherapist. Therefore, the TRQ should be applied at the start of treatment in order to identify relapse styles.

Although the TRQ among the study sample was found to be reliable and valid, reporting one general time to relapse score is better than reporting scores for the different subscales because various cross loadings were found between the subscales. This recommendation is similar to those of the developers of the original English TRQ.

The present study had some limitations. Firstly, due to the inaccessibility and problems related to stigma in women, this study included only men who were dependent on drugs. Hence, corroboration of our findings with data from both female and male patients would lend additional credibility to the findings. Secondly, the age of the patients in this study was 18 to 78 years.
Future studies should recruit patients younger than 18 years. Additionally, using the original English version of the instrument during the translation process could have created reaction bias due to the cultural differences between the United States and Iran. Additional studies with patients from different sociocultural contexts and backgrounds might reveal interesting results. Another major limitation of this study was that drug users with psychiatric disorders were excluded. This limits the generalizability of our findings. Furthermore, we didn't follow up with participants to investigate the predictive validity of the scale. This might be a direction for future research. Furthermore, it might be interesting for future studies to examine evidence of the convergent and discriminant validity of the scores of the Persian version of the TRQ by examining associations with scores of other relevant measures, such as impulsivity or executive function.

In summary, the findings from this study demonstrated that the Persian version of the TRQ questionnaire is a reliable and valid scale for measuring time to relapse in Iranian patients with a drug dependency history.

\section{Authors' contribution}

MM designed the project, collected the data, and performed the statistical analyses. TP was the research supervisor. MK helped as a consultant in the study design and questionnaire and critically revised the final article together with KP. All authors read and approved the final manuscript.

\section{Acknowledgments}

We would like to thank all participants for their participation in this study, as well as the experts without whose support this study would not have been possible.

\section{Funding}

Funding for this study was provided by Kurdistan University of Medical Sciences.

\section{Declaration of interest}

The authors declare that they have no competing interests.

\section{References}

1. Mokri A. Brief overview of the status of drug abuse in Iran. Arch Iran Med 2002;5:184-190.

2. Raisdana F, Nakhjavani AG. The drug market in Iran. ANNALS Am Acad Political Social Sci 2002;582:149-166.

3. Alam-mehrjerdi Z, Abdollahi M, Higgs P, Dolan K. Drug use treatment and harm reduction programs in 
Iran: a unique model of health in the most populated Persian Gulf country. Asian J Psychiatry 2015;16:78-83.

4. United Nations Office on Drugs and Crime: 2005 World Drug Report VIA.

5. Nissaramanesh B, Trace $M$, Roberts $M$. The rise of harm reduction in the Islamic Republic of Iran. Beckley Foundation Drug Policy Programme, Briefing Paper 2005;8.

6. Razzaghi E, Rahimi A, Hosseini M, Chatterjee A. Rapid Situation Assessment (RSA) of drug abuse in Iran. Prevention Department, State Welfare Organization, Ministry of Health, IR of Iran and United Nations International Drug Control Program 1999.

7. Razzaghi E, Rahimi-Movaghar A, Hosseini M, Madani S, Chatterjee A. Rapid Situation Assessment of Drug Abuse in Iran Tehran: Iranian Welfare Organization and UNDCP. 1999. Available at: http://www.unodc. org/pdf/iran/publications/RSA2000SUMMARY.pdf.

8. Pashaei T, Razaghi OM, Foroushani AR, Tabatabaei MG, Moeeni M, Turner NE, Sharifi V, Shojaeizadeh D. Assessing the validity and reliability of the Farsi version of inventory drug-taking situations. Iran J Psychiatry 2013;8:80.

9. O'Brien CP. Anticraving medications for relapse prevention: a possible new class of psychoactive medications. Am J Psychiatry 2005;162:1423-1431.

10. O'Brien C, McLellan AT. Myths about the treatment of addiction. Lancet 1996;347:237-240.

11. Mohammadpoorasl A, Fakhari A, Akbari H, Karimi F, Arshadi Bostanabad M, Rostami F, Hajizadeh M. Addiction relapse and its predictors: A prospective study. J Addict Res Ther 2012;3:1.

12. Shirinbayan P, Rafiey H, Vejdani Roshan A, Narenjiha $\mathrm{H}$, Farhoudian A. Predictors of retention in methadone maintenance therapy: a prospective multi-center study. Sci Res Essay 2010;5:3231-3236.

13. Tatari F, Shakeri J, Nasiri A, Ghelichi L, Abdoli G. The Relapse Rates Of Addiction In Opioid Dependent Patients Under Maintenance Therapy By Naltrexone Refered To Kermanshah Rehabilitating-Treating Center. 2006.

14. Maarefvand M, Eghlima M, Rafiey H, Rahgozar M, Tadayyon N, Deilamizadeh A, Ekhtiari H. Community-based relapse prevention for opiate dependents: a randomized community controlled trial. Community Mental Health J 2015;51:21-29.

15. Hser Y-I, Evans E, Huang D, Anglin DM. Relationship between drug treatment services, retention, and outcomes. Psychiatric Serv 2004;55:767-774.

16. Beynon CM, McMinn AM, Marr AJ. Factors predicting drop out from, and retention in, specialist drug treatment services: A case control study in the North West of England. BMC Public Health 2008;8:149.
17. Pashaei T, Shojaeizadeh D, Foroushani AR, Ghazitabatabae M, Moeeni M, Rajati F, Razzaghi EM. Effectiveness of relapse prevention cognitive-behavioral model in opioid-dependent patients participating in the methadone maintenance treatment in Iran. Iran J Public Health 2013;42:896-902.

18. Schonfeld L, Peters R, Dolente A. SARA-Substance Abuse Relapse Assessment. Odessa, FL: Psychological Assessment Resources Inc; 1993.

19. Turner NE, Annis HM, Sklar SM. Measurement of antecedents to drug and alcohol use: Psychometric properties of the Inventory of Drug-Taking Situations (IDTS). Behav Res Ther 1997;35:465-483.

20. Miller WR, Harris RJ. A simple scale of Gorski's warning signs for relapse. J Studies Alcohol 2000;61:759-765.

21. Adinoff B, Talmadge C, Williams MJ, Schreffler E, Jackley PK, Krebaum SR. Time to Relapse Questionnaire (TRQ): A measure of sudden relapse in substance dependence. Am J Drug Alcohol Abuse 2010;36:140-149.

22. Gable RK, Wolf MB. Instrument development in the affective domain. Boston, MA: Kluwer Academic Publishers; 1993.

23. Fitzner K. Reliability and validity a quick review. Diabetes Educ 2007;33:775-780.

24. Bartko JJ. The intraclass correlation coefficient as a measure of reliability. Psychological Rep 1966;19:3-11.

25. Baumgartner TA, Chung $H$. Confidence limits for intraclass reliability coefficients. Meas Phys Educ Exercise Sci 2001;5:179-188.

26. Polit DF, Beck CT. The content validity index: are you sure you know what's being reported? Critique and recommendations. Res Nurs Health 2006;29:489-497.

27. Waltz CF. Measurement in nursing and health research (4 ed.). New York, NY: Springer Publishing Company; 2010.

28. Lawshe $\mathrm{CH}$. A quantitative approach to content validityl. Personnel Psychol 1975;28:563-575.

29. Lacasse Y, Godbout C, Series F. Health-related quality of life in obstructive sleep apnoea. Eur Respir J 2002;19:499-503.

30. Harrington D. Confirmatory factor analysis. USA: Oxford University Press; 2008.

31. Mueller RO. Basic principles of structural equation modeling: An introduction to LISREL and EQS. New York, NY: Springer-Verlag; 1996.

32. Kline R. Principles and practices of structural equation modeling. (2nd ed.). New York: The Guilford Press; 2005.

33. Schumacker RE, Lomax RG. A beginner's guide to structural equation modeling. Psychology Press; 2004. 